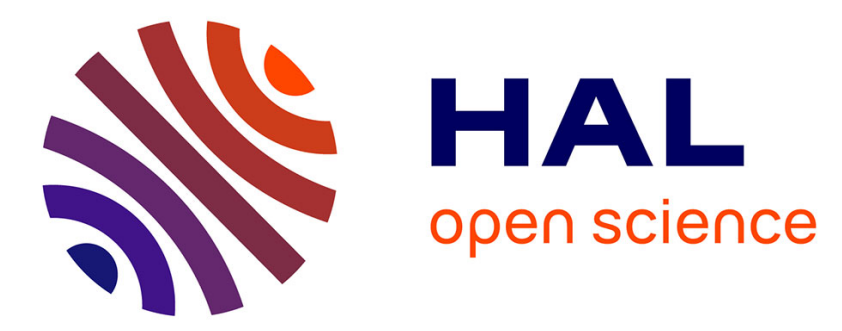

\title{
A Rat Tail Model for Osseointegration Studies and New Bone Formation Follow-up
}

Matthieu Renaud, Sandor Farkasdi, Alban Desoutter, Gabor Varga, Frédéric Cuisinier, Philippe Bousquet

\section{- To cite this version:}

Matthieu Renaud, Sandor Farkasdi, Alban Desoutter, Gabor Varga, Frédéric Cuisinier, et al.. A Rat Tail Model for Osseointegration Studies and New Bone Formation Follow-up. Journal of Cell Science \& Therapy, 2016, 7 (3), 10.4172/2157-7013.1000244 . hal-02046109

\section{HAL Id: hal-02046109 \\ https://hal.umontpellier.fr/hal-02046109}

Submitted on 1 Jun 2021

HAL is a multi-disciplinary open access archive for the deposit and dissemination of scientific research documents, whether they are published or not. The documents may come from teaching and research institutions in France or abroad, or from public or private research centers.
L'archive ouverte pluridisciplinaire HAL, est destinée au dépôt et à la diffusion de documents scientifiques de niveau recherche, publiés ou non, émanant des établissements d'enseignement et de recherche français ou étrangers, des laboratoires publics ou privés.

\section{(c)(1)}

Distributed under a Creative Commons Attribution| 4.0 International License 


\title{
A Rat Tail Model for Osseointegration Studies and New Bone Formation Follow-up \\ Matthieu Renaud ${ }^{1 *}$, Sandor Farkasdi ${ }^{2}$, Alban Desoutter ${ }^{1}$, Gabor Varga ${ }^{2}$, Frédéric Cuisinier ${ }^{1}$ and Philippe Bousquet ${ }^{1}$
}

${ }^{1}$ Laboratory Bioengineering and Nanoscience, University of Montpellier, Montpellier, France

${ }^{2}$ Department of Oral Biology Laboratory, Semmelweis University, Budapest, Hungary

\begin{abstract}
Animal studies are necessary to precede clinical studies. Recently the rat tail model has been proposed by us as a model for studying bone regeneration with easily surgical approach, effective control of post-operative pain and a decrease of animal number. The present study aimed to widen the rat tail model indication to implant osseointegration. Special titanium implants were inserted through tail vertebrae. A good primary stability was observed three month after implant placement. X-ray microtomography (Micro-CT) and histology were used to visualize bone formation and to calculate bone implant contact. Micro-CT showed osseointegrated implants in caudal vertebra. This illustrates the possibility to obtain bone implant-contact by micro-CT measurements. The results suggest that the rat caudal vertebrae may serve as a good preclinical model for studying implant osseointegration with the possibility of multiple testing within the same experimental animal and the potential to decrease number of experimental animals.
\end{abstract}

Keywords: Rat tail; Osseointegration; Bone formation; Micro-CT

\section{Introduction}

Animal studies are complementary to clinical experiments and must be used to prove the safety and efficacy of new medical devices. Many different animal models have been used to study bone regeneration or implant osseointegration $[1,2]$.

Desirable scientific attributes, particularly a relative similarity to human, must be defined clearly prior to select the animal. Favorable animal selection factors also include cost to acquire and care for animals, availability of the species, acceptability to society, tolerance to captivity and ease of housing [3]. Rodents such as mice, rats, and hamsters have been used widely for bone research because of small size, low cost, known age and genetic background, and ease of handling and housing [4]. Rat models, because of the larger animal size compared to mouse, are suitable to assess bone regeneration providing sufficient statistical significance reached by using numerous animals and to provide pre-clinical relevance [2]. Several sites (calvaria, tibia, femora, mandibular) have been used in various studies to investigate the effectiveness in bone defect repair of bone regenerative agents $[5,6]$. Unfortunately, none of these models combine surgical access facilities, number of defect sites and histological relevance.

2D histologic analysis has been traditionally used to assess the outcome of the experiment. X-ray micro-computed tomography (Micro-CT) is a powerful tool to asses bone healing [1]. Micro-CT also allows determining bone density [7].

Recently we have proposed a new rat model using caudal vertebrae [3]. This model can be used to follow bone formation with or without bone grafting materials. Moreover, up to four vertebrae per animal can be used. This is a key issue allowing diminishing the number of animals included in a given experiment due to multiple comparisons within one animal. This fact also increases the ethical acceptability of the new model [8]. Moreover, this model can be used to study osseointegration of alloplastic implant into caudal vertebrae. Live animals can be easily monitored by micro-CT to investigate for bone implant contact and bone formation in the vicinity of implants.

\section{Materiel and Methods}

\section{Experimental setup}

The study was approved by the committee for animal welfare of Montpellier University with the referral number 1083 16/06/2014. One group of ten male Wistar rats ((Crl:(Wi)Br) from Charles River France) with weight ranging from 380-450 $\mathrm{g}$ were used for an adequate vertebrae size. Implantation time was three months.

\section{Surgical procedure}

Animals were anesthetized with an intraperitoneal injection of ketamine and xylazine (Alcyon, Pau, France) ( 40 and $9 \mathrm{mg} / \mathrm{kg}$, respectively). A dorsal incision was performed approximately from $\mathrm{Cd} 31-\mathrm{Cd} 35$ vertebrae. The skin and the muscles were retracted and the vertebrae were exposed. Critical size defect drillings were performed with a 2.9 $\mathrm{mm}$ diameter drill through the center of the vertebrae. Impacted-type titanium $\left(\mathrm{Ti}_{6} \mathrm{Al}_{4} \mathrm{~V}\right)$ implants manufactured by FullTech Ltd (Budapest, Hungary) with a $2.9 \mathrm{~mm}$ wide coronal part were placed. After this procedure, the muscles and the skin were repositioned and sutured with resorbable sutures (Vicryl 4/0, Ethicon, Issy les Moulineaux, France). Following surgery, Buprenorphine SR-LAB (1 mg.mL $\left.{ }^{-1}\right)$, (Wildlife Pharmaceuticals, Windsor, CO, USA) at dose of $1.2 \mathrm{mg} \cdot \mathrm{kg}^{-1}$ was used for systemic relief and $72 \mathrm{~h}$ analgesia.

Rats were kept in individual cages and the wound healing was controlled daily during the first week and twice per week during the following healing period. Every second day the tails were disinfected using povidone-iodine solution (Betadine, Mundipharma, Paris, France).

\section{Sample collections}

At the end of the experimental periods, the rats were sacrificed by intraperitoneal injection of Pentothal (Alcyon, Pau, France) with a suitable dosage $(200 \mathrm{mg} / 1.5 \mathrm{~kg})$. The interested vertebrae were harvested. The samples were fixed in $5 \%$ formaldehyde solution in $4^{\circ} \mathrm{C}$ for 24 hours [9].

*Corresponding author: Renaud $\mathrm{M}$, Laboratory Bioengineering and Nanoscience EA 4203, University of Montpellier, 545 Avenue du Professeur Jean-Louis Vial, 34193 Montpellier Cedex, France, Tel: +33 6625287 81; E-mail: matt.renaud18@live.fr

Received: April 18, 2016; Accepted: June 24, 2016; Published: June 27, 2016

Citation: Renaud M, Farkasdi S, Desoutter A, Varga G, Cuisinier F, et al. (2016) A Rat Tail Model for Osseointegration Studies and New Bone Formation Follow-up. J Cell Sci Ther 7: 244. doi:10.4172/2157-7013.1000244

Copyright: @ 2016 Renaud M, et al. This is an open-access article distributed under the terms of the Creative Commons Attribution License, which permits unrestricted use, distribution, and reproduction in any medium, provided the original author and source are credited. 
Citation: Renaud M, Farkasdi S, Desoutter A, Varga G, Cuisinier F, et al. (2016) A Rat Tail Model for Osseointegration Studies and New Bone Formation Follow-up. J Cell Sci Ther 7: 244. doi:10.4172/2157-7013.1000244.

\section{Micro-CT analysis}

Vertebrae with osseointegrated implant were evaluated using an X-ray micro-CT instrument SKYSCAN 1176 X-ray Microtomograph (Bruker, Belgium). Implanted samples were scanned at $360^{\circ}$ rotation at 0.7 degree intervals. Measurements were made on the region of interest $(\mathrm{ROI}) \times 1.5 \mathrm{~mm}$ tissue volume $(\mathrm{TV})$ on the computer-reconstructed $3 \mathrm{D}$ samples. The gray scale images were reconstructed from the software Nrecon (Bruker, Belgium) and visualize with FIJI 1.5 software (NIH, USA). Then, these images were analyzed and aligned along the axis of the implant with DataViewer 1.5.2.4 software (Bruker, Belgium). The BIC analysis was performed with CTanalyser 1.15.4.0 software (Bruker, Belgium) and the visualization of the mesh with MeshLab 1.3.4 (INRC, Italy) and FIJI (NIH, USA) software.

\section{Histology}

After micro-CT scanning, the pieces were dehydrated in a series of graded ethanol solutions and embedded in epoxy resin blocks (Epoxy embedding medium kit, Sigma-Aldrich, France). Transverse sections were stained with toluidine blue.

\section{Results}

During experimentation, no infection of the operative site was observed. Every implant was osseointegrated. Rat behaviors did not change during the experimental and the post-surgical care periods.

Figure 1 shows the surgical set-up of the caudal vertebrae. After skin and muscles retraction a drilling was performed with a proper drill and an implant (Figure 1D) was impacted into the vertebrae (Figure 1B).

Three month after implantation, micro-CT scanning showed the implant position through the vertebra (Figure 2). Figure 2B demonstrates the implant after image treatment removing metal artifacts. Artifacts are removed by bean hardening correction, providing axial cross-section. Figure $2 \mathrm{C}$ shows $3 \mathrm{D}$ reconstruction of vertebrae showing presence of bone around the implant impacted.

In Figure 3 vertebra reconstruction shows presence of bone around

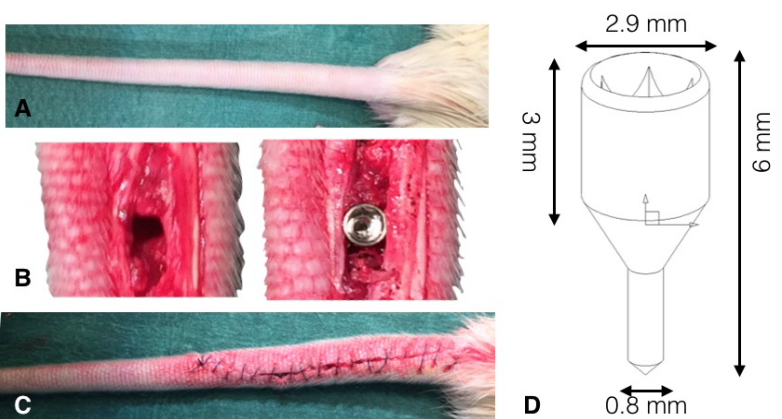

Figure 1: Surgical procedure. (A) Preoperative view of the rat tail. (B) Drilling position on the center of the vertebrae and implant placement after skin and muscle retraction. (C) Sutures. (D) Design of the implant.
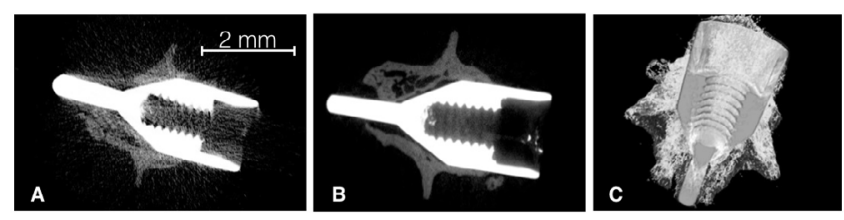

Figure 2: Micro-CT reconstruction of the interest rat caudal vertebra. (A) Initial reconstruction of vertebrae crossed by implant. (B) Beam hardening artifact treatment. (C) 3D reconstruction.
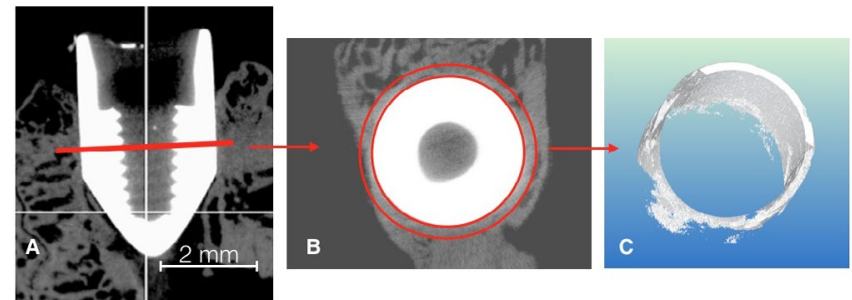

Figure 3: Bone implant contact measurement. (A) Transversal view, the red line indicates the interest plan. (B) Definition of ring (in red) around the area of interest. (C) 3D reconstruction of bone implant-contact (BIC).

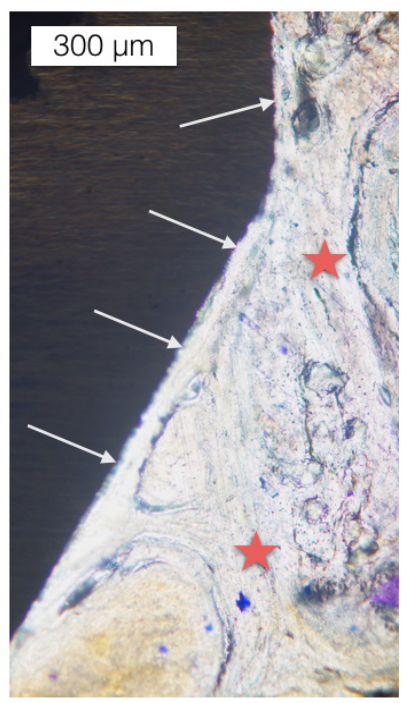

Figure 4: Histology showing implant osseointegration after 3 months. Gray arrows show the bone implant contact and red star show the new trabecular bone.

implant three month after the surgical procedure. Reference plan was defined on the neck of the implant (Figure 3A). After that, a ring was established around the reference area (Figure $3 \mathrm{~B}$ and $3 \mathrm{D}$ ) bone implant contact is presented in Figure 3C. Bone implant contact was calculated from these data and they gray scale pixels to obtain the quantity and the percentage of bone implant contact. Bone implant contact (BIC), percentage of BIC along the threads of the implant surface; and bone volume fraction/density; percentage of the peri-implant bone volume to the total could be calculated. Percent of bone implant contact is obtained dividing total intersection surface by tissue surface. In this situation, calculation of BIC represents 54.98 percent.

The histology analysis confirmed this osseointegration and showed bone-implant contact by a lot of trabecular bone in contact with titanium three months after implantation (Figure 4).

Micro-CT and histology analyses showed osseointegration by bone implant contact after three months.

\section{Discussion}

The present results validate our original assumption that the rat caudal vertebrae may serve as a good model for bone reconstruction. Consequently, various implant materials, surface treatment and surgical protocol can be studied using this rat tail model.

The best animal models should use the minimum number of animals providing reliable results. The possibility to use several vertebrae 
in the same animal gives a chance to decrease the number of rat using, for example, the same animal as proper control [3]. From a statistical point of view, one vertebra can be used for the control site and the others for different kind of experiments to compare them on the same rat at the same experimental time.

Another advantage of this new rat model is that the new surgical approach provides an easily, reproducible and, accessible site to perform the implant placement and, to control post-operative time. For histological and anatomical points of view, the caudal vertebrae of the rat are similar to human jawbones with abundant cancellous bone delimited with an important cortical bone thickness $[2,10]$. Moreover, caudal vertebrae of the rat are highly similar by the lack of hematopoiesis [10], a feature which is different in other bones which are frequently used as implant bed in animal models. This fact makes it a perfect model for evaluating implant osseointegration in dental and maxillofacial research in preclinical implant studies [11]. Many studies have shown the difficulty to experiment implant osseointegration with rat model because several negative points. The quality of the jawbones and a little size of the site are arguments avoiding using rat as model for implant [12]. These problems could be resolved with the rat vertebrae with an easily accessible site, safe and reproducible surgical procedure.

Indeed, following implant osseointegration, newly bone formation, bone implant-contact or, build kinetic model of healing, are a possibility with this model. Moreover, the titanium implant are specifically designed to be impacted into the cavity to obtain primary stability (coronal part is $2.9 \mathrm{~mm}$ wide), and to preserve a space between bone and implant in its apical part $(0.8 \mathrm{~mm}$ in diameter). The space between the apex-part can be left empty or be filled with biomaterial allowing bone implant-contact experiments in function of the type of biomaterials employed [3].

Micro-CT analysis may serve a reproducible possibility to calculate bone density and to assess bone remodeling and bone implant contact with simple procedure [7]. Unfortunately, artifacts are a problem for micro-CT analysis because they tend to under estimate the bone volume. Furthermore, methods are available to reduce or remove artifacts around the implant playing with variation of combined filters (copper/aluminum) or scan 360 . Bone implant contact is often cited in the literature like a validated method to calculate the quantity of bone in contact. A study compares the BIC with histological analysis and shows similar results with no-significant difference [13]. Studies showed BIC seems to be similar using special implant design into rat tibia in comparison with the rat vertebrae [14]. Moreover, studies demonstrated BIC seems to be also similar in clinical situation [15]. This model may become a very useful setting at preclinical level for both implantation and osseointegration studies.

\section{Conclusion}

The rat tail vertebrae model is promising for implant osseointegration studies. The model also permits adapted design implant placements with stability and, without pain and suffers. Micro-CT analysis permits to obtain bone implant contact by bone density calculation. This method can allow building a kinetic using iivo micro-CT and, only on the same animal. For ethical point of view, using this model is also an advantage by decrease the number of animals involved in the study.

\section{Acknowledgments}

This work was supported by the French-Hungarian TET-Balaton Program (TET_12_FR-2-2014-0010).

\section{References}

1. Ebina H, Hatakeyama J, Onodera M, Honma T, Kamakura S, et al. (2009) Micro-CT analysis of alveolar bone healing using a rat experimental model of critical-size defects. Oral Dis 15: 273-280.

2. Bhardwaj A, Bhardwaj SV (2012) Contribution of Animal Models in Periodontal Research. Int J Agro Vet Med Sci 6: 150-157.

3. Renaud M, Farkasdi S, Pons C, Panayotov I, Collart-Dutilleul PY, et al. (2015) A New Rat Model for Translational Research in Bone Regeneration. Tissue Eng Part C Methods.

4. Boix D, Weiss P, Gauthier O, Guicheux J, Bouler JM, et al. (2006) Injectable bone substitute to preserve alveolar ridge resorption after tooth extraction: a study in dog. J Mater Sci Mater Med 17: 1145-1152.

5. Espitalier F, Vinatier C, Lerouxel E, Guicheux J, Pilet P, et al. (2009) A comparison between bone reconstruction following the use of mesenchymal stem cells and total bone marrow in association with calcium phosphate scaffold in irradiated bone. Biomaterials 30: 763-769

6. Kummari SR, Davis AJ, Vega LA, Ahn N, Cassinelli EH, et al. (2009) Trabecular microfracture precedes cortical shell failure in the rat caudal vertebra under cyclic overloading. Calcif Tissue Int 85: 127-133.

7. Al-Hezaimi K, Ramalingam S, Al-Askar M, ArRejaie AS, Nooh N, et al. (2016) Real-time-guided bone regeneration around standardized critical size calvarial defects using bone marrow-derived mesenchymal stem cells and collagen membrane with and without using tricalcium phosphate: an in vivo microcomputed tomographic and histologic experiment in rats. Int J Oral Sci 8: 7-15.

8. Hartung T (2010) Comparative analysis of the revised Directive 2010/63/EU for the protection of laboratory animals with its predecessor 86/609/EEC - a t4 report. ALTEX 27: 285-303.

9. Blazsek J, Dobó Nagy C, Blazsek I, Varga R, Vecsei B, et al. (2009) Aminobisphosphonate stimulates bone regeneration and enforces consolidation of titanium implant into a new rat caudal vertebrae model. Pathol Oncol Res 15: 567-577.

10. Blazsek I, Goldschmidt E, Machover D, Misset JL, Benavides M, et al. (1986) Excess of lympho-reticular cell complexes in the bone marrow linked to $\mathrm{T}$ cell mediated dysmyelopoiesis. Biomed Pharmacother 40: 28-32.

11. Ysander M, Brånemark R, Olmarker K, Myers RR (2015) Intramedullary osseointegration: development of a rodent model and study of histology and neuropeptide changes around titanium implants. J Rehabil Res Dev 38: 183-190.

12. Struillou $X$, Boutigny $H$, Soueidan A, Layrolle $P$ (2010) Experimental animal models in periodontology: a review. Open Dent J 4: 37-47.

13. Bernhardt R, Kuhlisch E, Schulz MC, Eckelt U, Stadlinger B (2012) Comparison of bone-implant contact and bone-implant volume between 2D-histological sections and 3D-SR HCT slices. Eur Cell Mater 23: 237-247.

14. Pimentel SP, Casarin RC, Ribeiro FV, Cirano FR, Rovaris K, et al. (2016) Impact of micronutrients supplementation on bone repair around implants: microCT and counter-torque analysis in rats. J Appl Oral Sci 24: 45-51.

15. Salomó-Coll O, Maté-Sánchez de Val JE, Ramírez-Fernandez MP, HernándezAlfaro F, Gargallo-Albiol J, et al. (2015) Topical applications of vitamin D on implant surface for bone-to-implant contact enhance: a pilot study in dogs part II. Clin Oral Implants Res. 